\title{
Effect of microbial activity on dimethyl sulfoxide degradation in an air-lift bioreactor
}

\author{
S.-C. J. Hwang ${ }^{1}$, Y.-H. Lin ${ }^{2}$, Y.-C. Chang ${ }^{1} \&$ S.-Y. He ${ }^{1}$ \\ ${ }^{I}$ Department of Civil Engineering, Chung Hua University, Taiwan \\ ${ }^{2}$ Green Energy \& Environment Research Laboratories, \\ Industrial Technology, Taiwan
}

\begin{abstract}
Acclimatized dimethyl sulfoxide-degrading sludge can possess the ability to convert DMSO to odourless compounds specifically by repeated batches in an air-lift bioreactor. Moreover, DMSO usually causes a low microbial biomass growth, unstable operation and malodorous compounds emission in biological treatment because of its characteristic for activated sludge. A main focus of DMSO degrading performance is conducted by original sludge without longterm acclimation using an airlift bioreactor in this study. The result indicates various organisms of sludge poisoned by DMSO still secrete inducible oxidoreductase to improve volatile organic sulphur compounds degradation using DMSO as a substrate in an air-lift bioreactor. Using this method, the oxidoreductase in the supernatant of working medium is able to remove DMSO concentration from 50 up to $1000 \mathrm{mg} \mathrm{L}^{-1}$ completely by fed batch mode due to LB medium cultures and enhances the microbial activity of activated sludge. An optimal alternative performance was developed; the rate of DMSO biodegradation can accomplish $34.72 \mathrm{mg}$-DMSO $\mathrm{h}^{-1} \mathrm{~g}^{-1}$-dry sludge weight. The best result showed that $500 \mathrm{mg} \mathrm{L}^{-1}$ of DMSO could be completely removed with $120 \mathrm{~h}$ under repeated mode. Control of microbial activity in high loading of DMSO and carbon content in wastewater were important factors for biodegradation of DMSO.
\end{abstract}

Keywords: DMSO, VOCs, activated sludge, biodegradation, airlift bioreactor. 


\section{Introduction}

Dimethyl sulfoxide, or DMSO, is a good substitute for many organic solvents popularly used in a variety of industries. It has been widely used in the electronics industry, organic synthesis, oil extraction, synthetic fiber, etc. However, DMSO could be biodegraded under anaerobic conditions through the reduction pathway to produce malodorous DMS minute amounts of which could cause major complaints by the adjacent residents or inside-park factory workers. In Taiwan, many previous research results revealed that odorous DMS compound was mainly transformed from DMSO used tremendously in TFTLCD manufacture [1].

Degradation of DMSO used the efficiency of acclimated sludge by biological treatment in an airlift bioreactor was described in our previous research [2].

Many authors reported that control of the $\mathrm{pH}$ value was an important operating factor in biological treatment, impact of environmental odours by residual concentration, low microbial of biomass growth, or unstable operation, etc $[3,4]$. A variety of bacteria are able to degrade DMS and/or DMSO by oxidation or reduction in microbial cycling of sulfur compounds. Microorganisms have been isolated from sewage treatment plants, marine, sediments, soil, and biofilters. However, it needs to induce bacteria enzyme by organic or inorganic sulfur compounds because the specific degradation pathway of each substrate with selective cleavage of carbon-sulfur bonds by monooxygenases.

In our previous paper showed that an airlift bioreactor can supply oxygenenriched of operation condition. The reaction time could further be shortened to less than $10 \mathrm{~h}$ with $95 \%$ removal of the $750 \mathrm{mg} \mathrm{L}-1 \mathrm{DMSO}$ at the maximum rate of $0.909 \mathrm{~g}$ DMSO per gram suspended solids per day using an oxygen-enriched air-lift bioreactor and no malodorous compounds, such as dimethyl sulfide, were produced from DMSO biodegradation [2]. By treating 1,200 $\mathrm{mg} \mathrm{L}^{-1}$ of DMSO required more than $45 \mathrm{hr}$ for complete biodegradation, compared to treating $800 \mathrm{mg} \mathrm{L}^{-1}$ of DMSO only requiring $21 \mathrm{hr}$ for complete degradation using PVA immobilized sludge beads in an airlift bioreactor [5].

For biological treatment in wastewater treatment, chemical treatment plant or sewage treatment plant, search specific sludge or isolate useful bacteria can definitely economize cost and save time to process wastewater systems. To face the toxicity of DMSO and use existing methods to improve the inhibition of DMSO biodegradation is a first subject. Therefore, the main goal of this paper in using an airlift bioreactor we performed a series of improvements from unacclimatized activated sludge and observed the condition of unspecific sludge on degradation of DMSO by complex composition of source. Some influencing factors including acclimation, working medium, addition of sucrose and reaction of toxicity in DMSO degradation were examined. 


\section{Materials and methods}

\subsection{Microorganisms and cultivation}

According to our previous report, the aerobic activated sludge which was newly obtained from a chemical plant (Chang Chun Group, Miaoli County, Taiwan) was chosen as an inoculum. It was not incubated with a medium and fed 600-mg $\mathrm{L}^{-1}$ of DMSO as a substrate to acclimate the sludge in this study. The composition of working medium was described by Yang and Myint [6] and all chemicals were obtained from Merck \& Co. Inc. (Whitehouse Station, USA). All other chemicals were of reagent grade or above.

\subsection{Airlift bioreactor}

A schematic diagram of the airlift bioreactor is shown in Figure 1. The airlift bioreactor was built using an annular acrylic column with a height of $47 \mathrm{~cm}$ and internal diameters (ID) of $11 \mathrm{~cm}$ and $5 \mathrm{~cm}$ for an external and internal column, respectively, in which there was a $33.5 \mathrm{~cm}$ long drag tube. Approximately $350 \mathrm{ml}$ of pre-cultured inoculums with the suspended solid concentration of around $25,000 \mathrm{mg} \mathrm{L}^{-1}$ was added the airlift bioreactor, before the TA-1 medium was filled up to $3.2 \mathrm{~L}$. The liquid was circulated by the input air flow rate of $10 \mathrm{~L}$ $\min ^{-1}$ with Hiblow pump (Sun Mines, Taipei County, Taiwan).

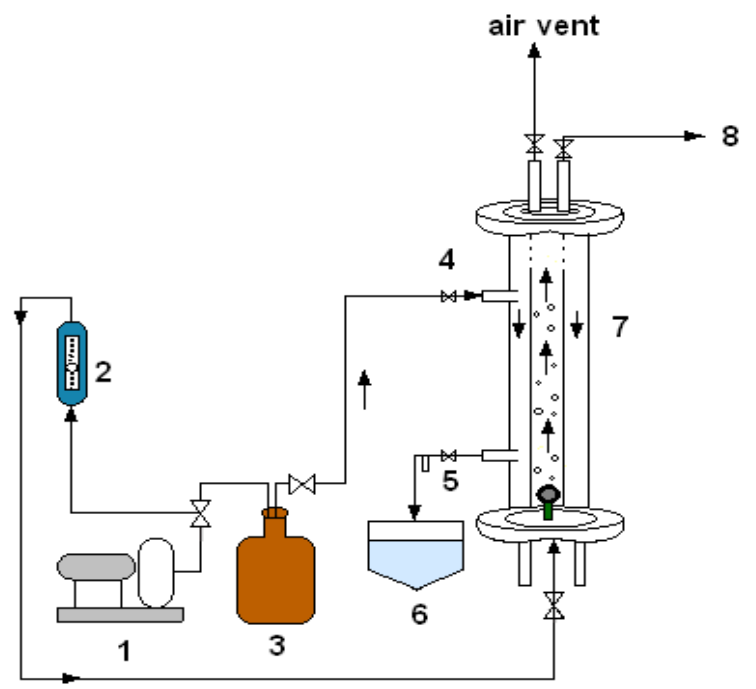

Figure 1: Set-up of air-lift system: (1) diaphragm air pump; (2) rotameter; (3) DMSO reservoir; (4) feeding port; (5) liquid sampling port; (6) settler; (7) airlift; (8) gas sampling port for DMS using Tedlar bags. 


\subsection{Experimental design}

According to our previous report, DMSO degradation needs an appropriate acclimation time to allow the mono-oxygenized enzymes being synthesized [2]. Therefore, a fresh and new DMSO-degrading culture of $300 \mathrm{~mL}$ of supernatant in working medium was used as inoculums for biodegradation of $500 \mathrm{mg}$ DMSO L ${ }^{-1}$ under the condition of whether or not adding sucrose as a carbon source (0 or $10 \mathrm{~g}$ of sucrose). In comparison of oxidoreductase degradation results, the new activated sludge culture was chosen to operate in an airlift bioreactor which can demonstrate optimal performance in biological treatment directly for biodegradation of DMSO.

\subsection{Analytical methods}

Two milliliters of water sample were filled into a 2-ml aliquot and centrifuged at $10,000 \mathrm{rpm}$ for $10 \mathrm{~min}$. After the supernatant was filtered out through a $0.22 \mu \mathrm{m}$ filter as the report described by Murakami et al. [7], a needle was employed to suck out $0.1 \mu \mathrm{L}$ of the filtered liquid for measuring DMSO concentration using high performance liquid chromatography (HPLC) with UV or RI detectors (Chromatograph Acme 9000, Younglin Instrument, Korea) equipped with a Hypersil ODS column $(5 \mu \mathrm{L}, 250 \times 4.6 \mathrm{~mm}$, England). The DMS gas was used gas chromatography/flame ionization detector (Chromatograph Acme 6000) equipped with a Quadrex 007-5 fused silica capillary column (25-m length, 0.25mm I.D., $0.5-\mu \mathrm{m}$ film) and bonded with 5\% phenyl methylpolysiloxane or DB-5. The temperature of the injector, oven, and detector were 90,100 , and $200^{\circ} \mathrm{C}$, respectively. The $\mathrm{pH}$ and optical density (OD) were measured using a $\mathrm{pH}$ meter and spectrophotometer at $600 \mathrm{~nm}$, respectively.

\section{Results and discussion}

\subsection{Microbial activity on DMSO biodegradation in airlift bioreactor}

In order to prove the inducible oxidoreductase exists in wide variety organisms for microbial cycling of DMSO and observe the toxicity of DMSO compound for unacclimatized activated sludge. The upper layer liquid of settled sludge was taken into the airlift bioreactor from $3000 \mathrm{~mL}$ of artificial wastewater medium which after induced by $50 \mathrm{mg} \mathrm{L}^{-1}$ of DMSO. The microbial activity of activated sludge on DMSO degradation by step loading of DMSO is shown in Figure 2. As shown, there was a rapid drop trend from the first four of fed batch by degrading DMSO in $50 \mathrm{mg} \mathrm{L}^{-1}$. The first step of trend reflected the oxidoredutase is inducible and can degrade DMSO totally by many times. Moreover, in the second step of degrading DMSO in $400 \mathrm{mg} \mathrm{L}^{-1}$, it was then a steady line after $100 \mathrm{~h}$. The result of the second step showed the inactive enzyme emerges in DMSO degradation by a sudden high concentration of DMSO and the toxicity remains a low biomass of microbial. The inactivity of enzyme still kept to third step even though the concentration gained $50 \mathrm{mg} \mathrm{L}^{-1}$ of DMSO. The phenomenon demonstrates that the inducible oxidoreductase existing in the upper layer liquid 

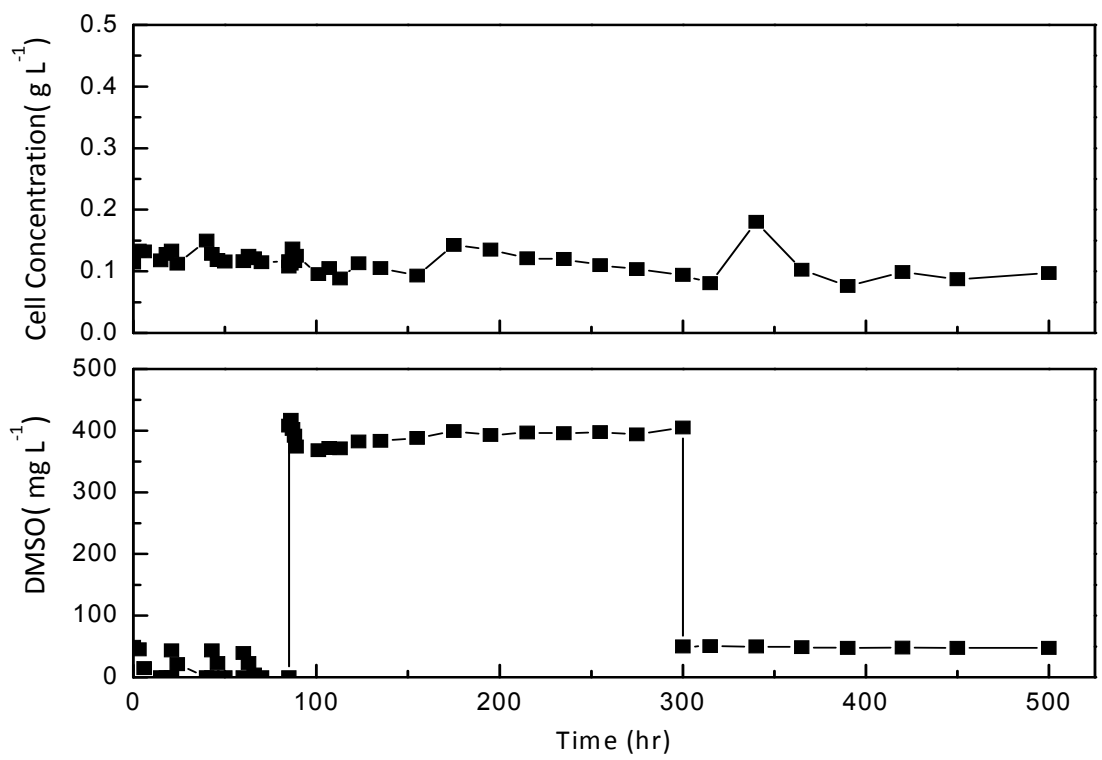

Figure 2: Microbial activity performance on DMSO degradation by step loading of DMSO.

can remove DMSO and the low biomass causes weak microbial activity to be inactive.

\subsection{Various working medium on DMSO biodegradation by fed mode performance}

According to Horinouchi et al. [8] reports that sulfur-free mineral medium (SFMM) was used to enrich and isolate for DMS-utilizing bacteria, including $10000 \mathrm{mg} \mathrm{L}^{-1}$ of carbohydrate, $3000 \mathrm{mg} \mathrm{L}^{-1}$ of nitrogen, 2.2 and $0.8 \mathrm{~g} \mathrm{~L}^{-1}$ of phosphate. Various compositions of working medium on DMSO degrading perform in Figures 3 and 4. From the Figure 3 it can be seen that $500 \mathrm{mg} \mathrm{L}^{-1}$ of DMSO is able to be handled in an airlift bioreactor using a SFMM working medium by fed batch and biomass has good microbial activity in sludge. The complex of wastewater sources sometimes causes environmental odor problems such as DMS or $\mathrm{H}_{2} \mathrm{~S}$ in a modern multiple wastewater system by the increase of BOD even with waste residues of DMSO [3]. As can be seen in Figure 4, it is out of activity after the third round by $500 \mathrm{mg} \mathrm{L}^{-1}$ loading of DMSO and low biomass appears in the end. The medium used in Figure 4 is from Yang and Myint [6], including $50 \mathrm{mg} \mathrm{L}^{-1}$ of carbohydrate, $120 \mathrm{mg} \mathrm{L}^{-1}$ of nitrogen, 2.72 and $5.23 \mathrm{~g} \mathrm{~L}^{-1}$ of phosphate. Although the result indicated that the best degradation time by $500 \mathrm{mg} \mathrm{L}^{-1}$ of DMSO reaches $144 \mathrm{~h}$, the biomass and microbial activity are low after $100 \mathrm{~h}$. The phenomenon represents that it has higher biomass and microbial activity can remove $500 \mathrm{mg} \mathrm{L}^{-1}$ of DMSO totally in Figure 3; this has a faster rate and is more specific on DMSO degradation but low biomass and microbial activity occurs. 


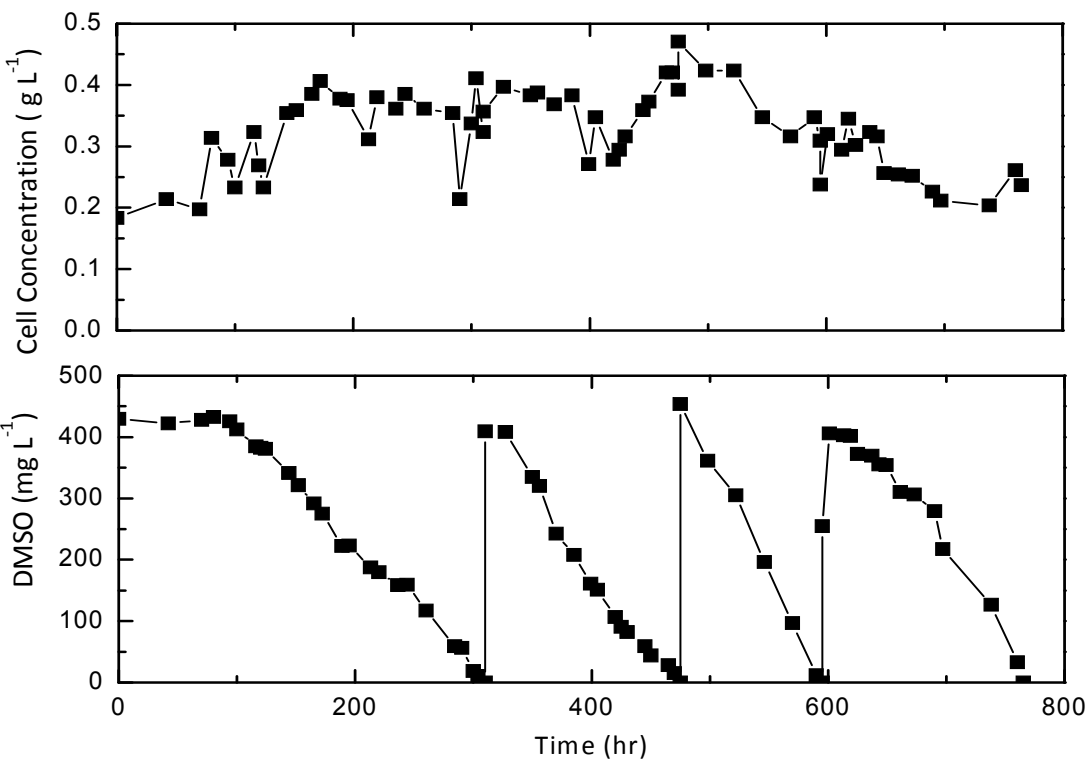

Figure 3: Degradation of DMSO by operating in sulfur-free medium modified (SFMM) working medium under a fed-batch mode.

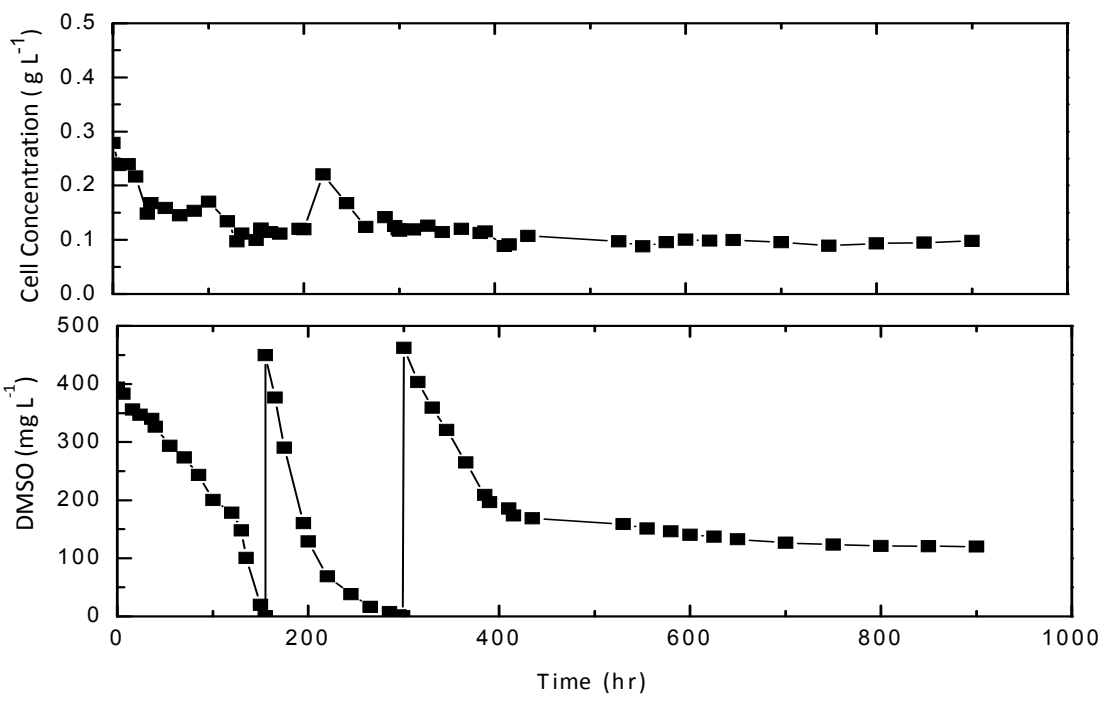

Figure 4: DMSO degrading by operating in TA-1 working medium under a fed-batch mode. 


\subsection{Effect of microbial activity on DMSO degradation by LB medium under a fed mode in airlift bioreactor}

In order to increase the microbial activity in sludge, the pre-culture is a key point for degradation. Before activated sludge started the experiments, the pre-culture using LB medium was carried out in DMSO biodegradation.

\subsubsection{Biodegradation of DMSO without culture in LB medium}

The microbial activity and biomass were investigated in sludge to realize the tolerance and degradation for sudden high loading DMSO. Figure 5 shows that microbial activity of activated sludge on DMSO biodegradation without preculture in LB medium which can completely degrade DMSO six times within $6 \mathrm{~h}$. The time for completing $200 \mathrm{mg} \mathrm{L}^{-1}$ of DMSO of the reaction increased from 220 to $300 \mathrm{~h}$, indicating that the microbial activity of activated sludge decreased gradually with low biomass. There are still a lot of limits for a company to handle wastewater continuously and stability because of microbial activity such as inadequate for microorganisms, unsupplied sufficient conditions of reactor, and unfriendly environment to destroy persistent DMSO chemical.
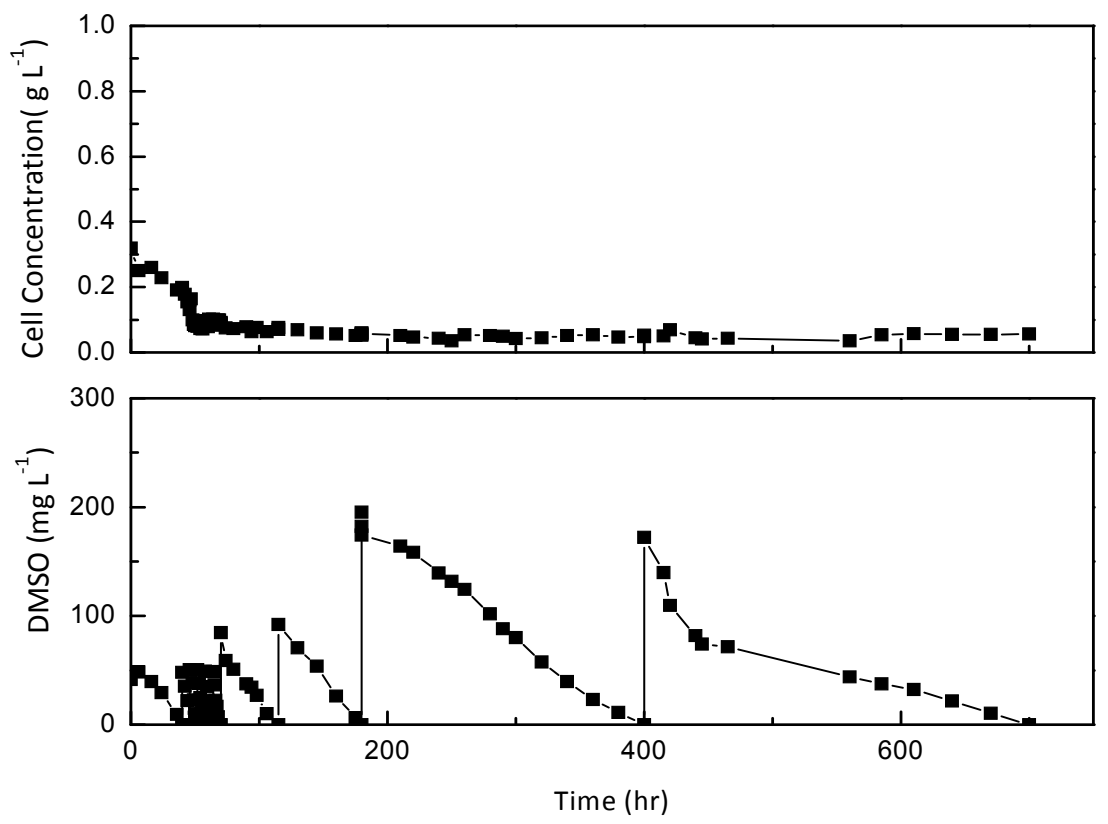

Figure 5: Microbial activity on DMSO degradation without pre-culture in LB medium.

\subsubsection{Degradation of DMSO with culturing in LB medium}

Figure 6 displays that microbial activity on DMSO degradation with pre-culture in LB medium can remove $500 \mathrm{mg} \mathrm{L}^{-1}$ of DMSO at $120 \mathrm{~h}$ by being operated five times. The reaction time of DMSO degradation decreased from 256 to $120 \mathrm{~h}$ by 
degrading $500 \mathrm{mg} \mathrm{L}^{-1}$, indicating that the microbial activity increased gradually by sudden loading high concentration of DMSO.

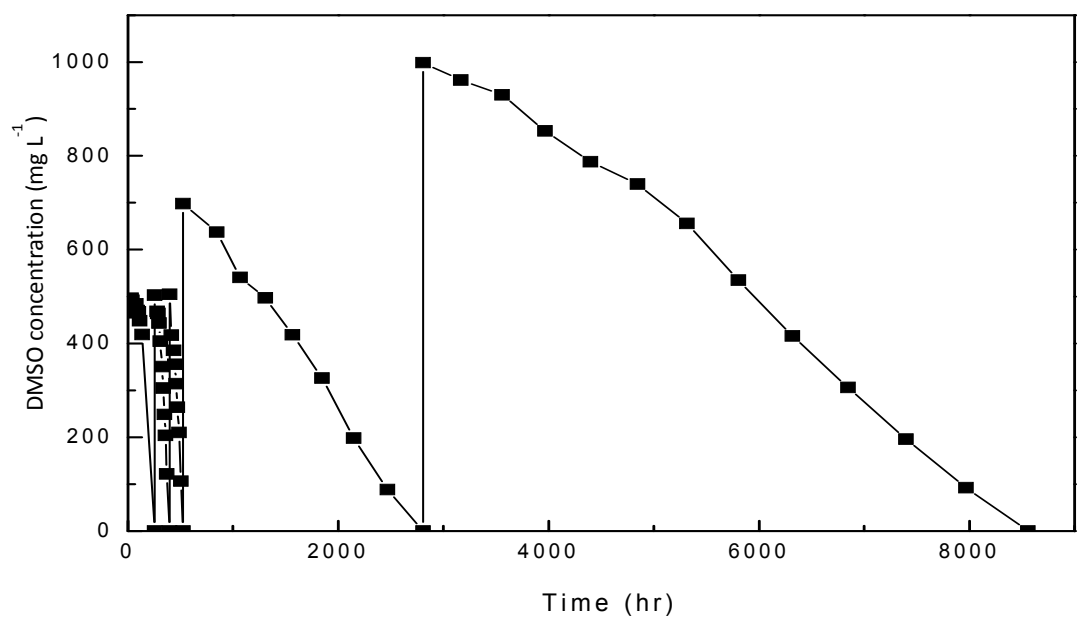

Figure 6: Microbial activity on DMSO degradation with pre-culture in LB medium.

\subsection{Effect of carbohydrate content on biodegradation of DMSO}

According to Yang's report [6], adding $50 \mathrm{mg} \mathrm{L}^{-1}$ of sucrose to the DMSOcontaining wastewater will enhance the biodegradation of DMSO. The TOC removal efficiency would increase from $80-84 \%$ to $92-95 \%$. The additional sucrose compound was the main cause to enhance the microbial capability in detoxification of the toxic DMSO. The similar reaction mechanism was also found in the co-metabolism [9]. The $500 \mathrm{mg} \mathrm{L}^{-1}$ of DMSO was biodegraded with various sucrose contents under repeated batches and the results are illustrated in Figure 7. The biodegradation of DMSO was not acclimated well, and it was inhibited so as to have a low removal efficiency of only $71.8 \%$ in $284 \mathrm{hr}$, when the sucrose content went up to $5000 \mathrm{mg} \mathrm{L}^{-1}$. Moreover, the addition of an extra carbon source seems to have a positive effect on the initial rates of biodegradation of DMSO. The largest initial rate occurred at a sucrose content of $10000 \mathrm{mg} \mathrm{L}^{-1}$, but the reaction rate quickly decreased possibly due to some intermediates produced from the sucrose. However, the reactions with 5000 and $10000 \mathrm{mg} \mathrm{L}^{-1}$ of DMSO were far slower than those with $0-50 \mathrm{mg} \mathrm{L}^{-1}$ of DMSO. This situation was demonstrated that activated sludge without acclimation is able to degrade DMSO specifically when using property carbon content in the experiment but it doesn't display well with the addition of a high content of carbon source. Obviously, the results showed almost $20 \mathrm{ppm}$ of the malodorous DMS was produced when sucrose went up to 50000 and $10000 \mathrm{mg} \mathrm{L}^{-1}$ of DMSO in the beginning. Although the DMSO degradation was completely removed from activated sludge, the composition of carbon content could affect the specific in sulfur metabolism. 


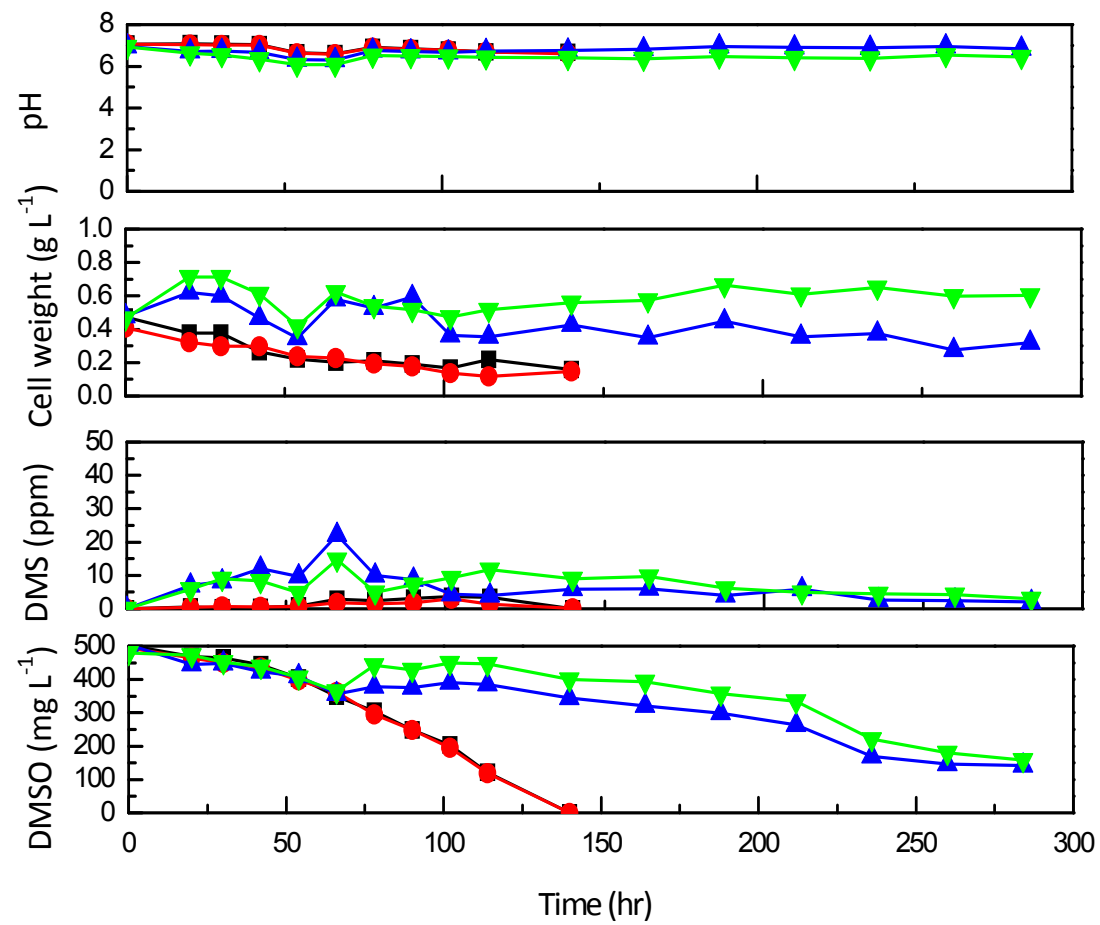

Figure 7: Effect of carbon source on degradation of DMSO in an airlift bioreactor. Initial sucrose concentration: (๘) $0 \mathrm{mg} \mathrm{L}^{-1}$; (•) $50 \mathrm{mg} \mathrm{L}^{-1}$; (४ ) $5000 \mathrm{mg} \mathrm{L}^{-1}$; ( $\left.\mathbf{\nabla}\right) 10000 \mathrm{mg} \mathrm{L}^{-1}$.

\subsection{Airlift performance in repeated batch mode}

In the suspended sludge system, the airlift performance was illustrated in Figure 8. Five hundred $\mathrm{mg} \mathrm{L}^{-1}$ of DMSO was removed in $48 \mathrm{hr}$ with the removal efficiency of near $99 \%$ by acclimation with the addition $50 \mathrm{mg}$ of sucrose. Muratani [10] has reported that $600-\mathrm{mg} \mathrm{L}^{-1}$ DMSO required more than $15 \mathrm{hr}$ for complete biodegradation. Yang and Myint [6] have also reported that $600-\mathrm{mg} \mathrm{L}^{-1}$ DMSO needed $6.66 \mathrm{hr}$ for $95 \%$ TOC removal. Using microbial activity characteristics, the airlift performance with many repeated batches was getting faster by enhancing in LB medium. Thus, the microbial activity of microorganisms in an airlift bioreactor could stand at double loading of DMSO. Znad et al. [11] adopted airlift with suspended activated sludge or immobilized cell beads at an air flow rate of $3.5 \mathrm{~L} \mathrm{~min}^{-1}$ under repeated batches to treat 2,184 ppm of S-ethyl dipropylthiocarbamate (EPTC). Using immobilized cell beads could avoid the toxicity of EPTC and its removal efficiency was higher than using the suspended activated sludge system. 


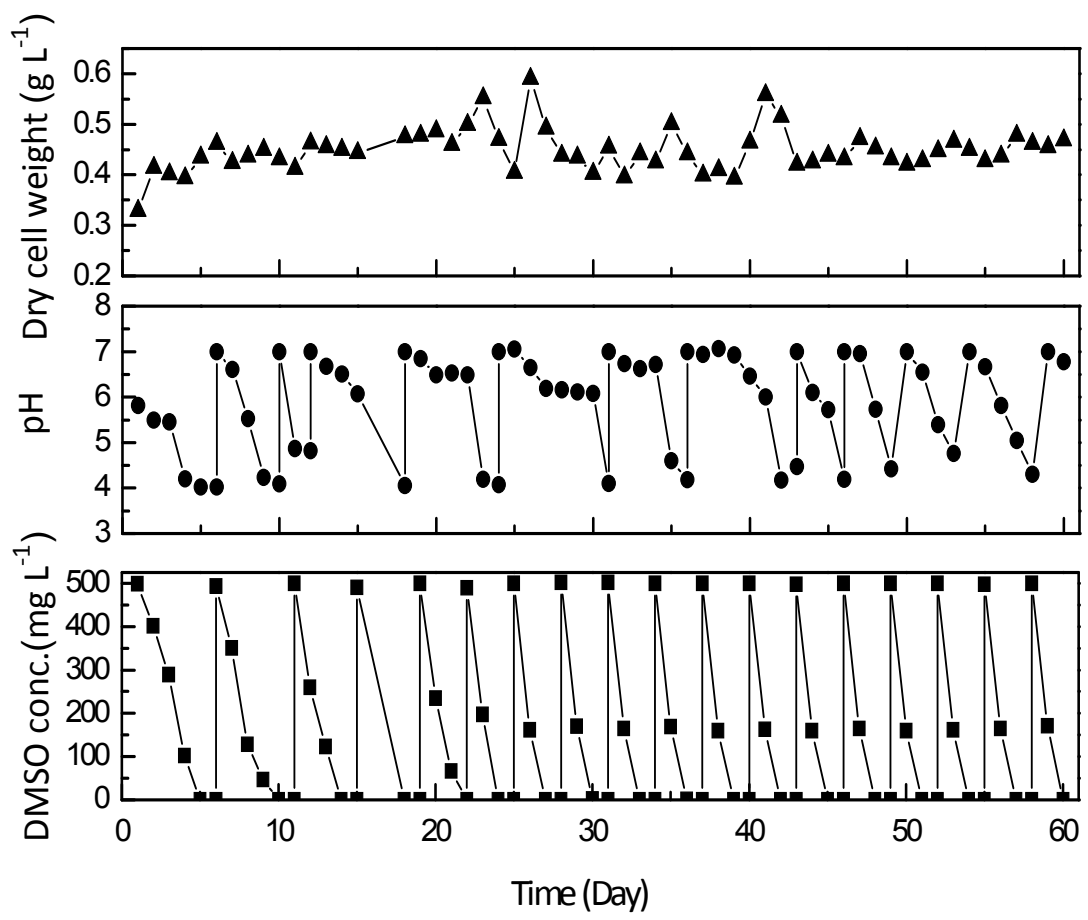

Figure 8: Microbial activity on degradation and acclimation of DMSO degradation by repeated batch mode in airlift bioreactor.

\section{Conclusions}

In a repeated-batch mode, the activated sludge system could completely degrade a lower dose of DMSO such as $50 \mathrm{mg} \mathrm{L}^{-1}$ of DMSO for many times. However, the biodegradation of DMSO was successfully treated only three times and then fully retarded when the concentration of DMSO was increased to $500 \mathrm{mg} \mathrm{L}^{-1}$. It was confirmed that the three times of successful degradation was achieved by the original existing enzyme after we separated the microorganisms and medium apart using centrifugation. Since the DMSO uptake couldn't increase the biomass of activated sludge and then no more enzyme was secreted for use. The better solution for the treatment of DMSO of $500 \mathrm{mg} / \mathrm{L}$ was to add the LB to the synthesis wastewater intermittently. The result showed the $500 \mathrm{mg} / \mathrm{L}$ of DMSO could be completely removed within $120 \mathrm{~h}$ under repeated mode all the way.

\section{References}

[1] Lin, Y.-C., Chang, F.-T., Bai, H., and Pei, B.-S., Control of VOCs emissions by condenser pre-treatment in a semiconductor fab. Journal of Hazardous Materials, 120(1-3), pp. 9-14, 2005. 
[2] Hwang, S.-C. J., Wu, J.-Y., Lin, Y.-H., Wen, I. C., Hou, K.-Y., and He, S.Y., Optimal dimethyl sulfoxide biodegradation using activated sludge from a chemical plant. Process Biochemistry, 42(10), pp. 1398-1405, 2007.

[3] Glindemann, D., Novak, J., and Witherspoon, J., Dimethyl Sulfoxide (DMSO) Waste Residues and Municipal Waste Water Odor by Dimethyl Sulfide (DMS): the North-East WPCP Plant of Philadelphia. Environmental Science \& Technology, 40(1), pp. 202-207, 2005.

[4] Park, S.-J., Yoon, T.-I., Bae, J.-H., Seo, H.-J., and Park, H.-J., Biological treatment of wastewater containing dimethyl sulphoxide from the semiconductor industry. Process Biochemistry, 36(6), pp. 579-589, 2001.

[5] He, S.-Y., Lin, Y.-H., Hou, K.-Y., and Hwang, S.-C. J., Degradation of dimethyl-sulfoxide-containing wastewater using airlift bioreactor by polyvinyl-alcohol-immobilized cell beads. Bioresource Technology, 102(10), pp. 5609-5616, 2011.

[6] Yang, P., and Myint, T., Integrating entrapped mixed microbial cell (EMMC) technology for treatment of wastewater containing dimethyl sulfoxide (DMSO) for reuse in semiconductor industries. Clean Technologies and Environmental Policy, 6(1), pp. 43-50, 2003.

[7] Murakami-Nitta, T., Kurimura, H., Kirimura, K., Kino, K., and Usami, S., Continuous degradation of dimethyl sulfoxide to sulfate ion by Hyphomicrobium denitrificans WU-K217. Journal of Bioscience and Bioengineering, 94(1), pp. 52-56, 2002.

[8] Horinouchi, M., Kasuga, K., Nojiri, H., Yamane, H., and Omori, T., Cloning and characterization of genes encoding an enzyme which oxidizes dimethyl sulfide in Acinetobacter sp. strain 20B. FEMS Microbiology Letters, 155(1), pp. 99-105, 1997.

[9] Rittman, B. E., and Mccarty, P. L., Environmental Biotechnology: Principles and Application. New York: McGraw-Hill Inc., 2001.

[10] Muratani, T., Biological treatment of wastewater containing DMSO. Sharp Giho, 73, pp. 20-25, 1999.

[11] Znad, H., Kasahara, N., and Kawase, Y., Biological decomposition of herbicides EPTC by activated sludge in a slurry bioreactor. Process Biochemistry, 41, pp. 1124-1128, 2006. 